\title{
Blockchain Network Model to Improve Supply Chain Visibility based on Smart Contract
}

\author{
Arwa Mukhtar ${ }^{1}$, Awanis Romli ${ }^{2}$, Noorhuzaimi Karimah Mohd ${ }^{3}$ \\ Faculty of Computing, Universiti Malaysia Pahang \\ Kuantan, Malaysia
}

\begin{abstract}
Due to the increasing complexity of supply chains over the past years, many factors significantly contribute to lowering the supply chains performance. Poor visibility is one of the major challenging factors that lowers supply chains performance. This paper proposes a Blockchain-based supply chain network model to improve the supply chain visibility. The model focuses in improving the visibility measurements properties: information sharing, traceability, and inventory visibility. The proposed model consists of information sharing, traceability, and inventory visibility platforms based on Blockchain technology smart contract. The model built with Hyperledger platform and extend the Hyperledger Composer Supply Chain Network (HCSC) model. The research is designed to three main phases. First phase: the preliminary phase which is the literature review phase to identify the existing challenges in the domain. The second phase: the design and implementation phase which is the development steps of the proposed research model. The third phase: the evaluation phase which represent the performance evaluation of the proposed model and the comparisons between the proposed model and the existing models. In the evaluation performance, the common performance metrics Lead time and average inventory levels will be compared in the proposed model, Cloud-based information system, and the traditional supply chain. These proposed platforms offer an end-to-end visibility of products, orders, and stock levels for supply chain practitioners and customers within supply chain networks. Which helps managers' access key information that support critical business decisions and offers essential criteria for competitiveness and therefore, enhance supply chain performance.
\end{abstract}

Keywords-Supply chain management; supply chain visibility; blockchain; smart contact; information sharing; traceability; inventory visibility

\section{INTRODUCTION}

Given the globalization and expansion in the supply chain operations, the complexity of chain operations have been increased [1-4]. Due to the decentralized nature of the supply chain, firms are unable to identify the origins of problems that arises within their supply chain flow [5]. For instance, the recall of millions of toys in Mattel (an American multinational toy manufacturing company) in 2015. The firm loss was over $\$ 110$ million for product return due to unauthorized paints in its product from an unauthorized supplier. Furthermore, more importantly though in health and food supply chain. Where demand uncertainties and long lead-times of medical supplies and drugs are critical to the whole process specially in health and food safety issues [6]. Moreover, lack of visibility is still a challenging issue for supply chain executives especially in increasingly complex environment [3, 7, 8]. Improving the visibility level in the supply chain is essential not only to gain access to key decision-making information [9], but also to facilitate more transparency and traceability within the supply chain network [10]. Moreover, in terms of inventory management, the visibility of product inventory reduces the stock repetition, the out of stock situations, and the inventory cost [3]. Overall, the poor visibility hinders efficient supply chain performance [8]. As a consequence, it is critical for firms that experiences such problems caused by poor visibility to adopt new innovative technologies and business models to overcome these challenges [11].

Recently, leading-edge firms using Blockchain technology to optimize their business processes, velocity, throughput, latency, compliance, cost-effective, provenance awareness, and building trust [12]. Blockchain technology has been used to enhance cost effectiveness and security of supply chain transactions [13], physical distribution visibility and shipment tracking [14], risk management [15]. Moreover, recent studies have been employed Blockchain technology to enhance supply chain visibility $[16,17]$. Blockchain technology appears as a good fit for digital supply chain integration because of its ledger, security and smart contract platforms, as well as software connectors $[13,18]$. Moreover, the technology offers tools to build a cost-effective and flexible digital supply chain network [19]. Thus, it is the right solution to solve core problems in supply chain like transparency, traceability, and poor visibility [17].

Therefore, in this paper, a Blockchain-based supply chain model is proposed to improve supply chain visibility using Blockchain smart contract. Smart Contracts can improve supply chain visibility of assets, orders, and inventory levels, therefore improves information sharing, order traceability and improve inventory visibility.

This paper is organized as follows: Section 2 introduces Blockchain technology and reviews the related literature of the technology. Section 3 reviews the supply chain visibility and explains how Blockchain technology can help improving the supply chain visibility. Section 4 describes the research agenda. While section 5 includes the description of the proposed model and its components. section 6 describes the evaluation phase of the proposed model. Finally, section 7 concludes the paper.

\section{BLOCKCHAIN TECHNOLOGY}

Blockchain technology was introduced as the core technology of Bitcoin, which is the first electronic payment 
system based on a decentralized peer-to-peer network [20]. Blockchain is a digital, shared, immutable, decentralized ledger technology that facilitates sharing transaction records between parties and grouped them into blocks chained to each other [13, 21]. These transactions records are stored in a shared, decentralized ledger without the need of any third-party intermediary $[22,23]$. Figure 1 shows the process of building the blocks.

Recently, beyond the Bitcoin, this emerging technology is used in many sectors such as in food manufacturing [24], environmental solution [25], energy sector [26], and supply chain [27]. In the last few years Blockchain technology has been utilized for non-financial applications [28]. Therefore, multiple Blockchain platforms have been developed [29, 30]. Hyperledger, is an open source permissioned Blockchain (Hyperledger Project,2015). While Bitcoin, Ethereum, Litecoin are examples of cryptocurrencies applications of public Blockchain [31].

Recent research communities have examined different aspects of Blockchain and its implication to the entire enterprises ecosystems. Many related perspectives has been investigated such as: security [15], privacy [32], data management Banerjee et al. (2017), identity management [33] and other majors explored and future research directions has been revealed apparently.

In supply chain context, Blockchain promotes the availability of information about the product to all the participating parties in the Blockchain network which offers an enhanced visibility throughout the product life cycle [15]. The registered transfer of goods and any change in the process products details will be documented in a decentralized database. This information availability ensures efficient transactions, efficient recalls in addition to the assurance of food safety and prevention of counterfeits [16]. The tracking and traceability system that Blockchain offers serves supply chains as a secure, transparent and traceable platform that facilitates tracking the materials and determine the provenance or origins of a product from its original to the end customers [34-36]. Since tracking and authenticating the product provenance or origins is critical to the food supply chain [37].

In the same context, both literature review and the practice revealed that using new emerging technologies such as Blockchain technology for the visibility issue seems promising $[14,16-18,30]$. Attention is being given to the benefits that Blockchain can offer for the supply chain, which lead the researchers to emphasis more on the potential capabilities of Blockchain in resolving supply chain challenges. Although prior studies developed different Blockchain-based solutions to increase the supply chains visibility [14, 16-18, 30], according to Dolgui, Ivanov [38], "There is still a lack of scientific methodology to Blockchain-driven design of the smart contracts in the supply chain.". In line with the literature review findings of Queiroz, Telles [39], "Smart contracts will contribute to several SCM improvements such as improved responsiveness, lead-time reduction, transaction costs reduction, increased visibility and more trust, security and transparency in the network".

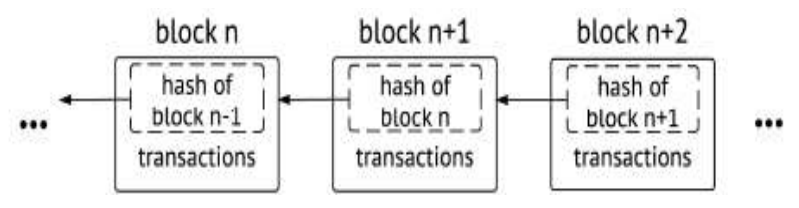

Fig. 1. The Building Blocks Process [23].

Therefore, the proposed HCSC-POS model in this paper differs from alternative models in using Blockchain smart contracts to increase the visibility measurements: information sharing, orders tracking and inventory visibility and aims to fill this gap in the literature.

\section{SUPPLy ChAIN VISIBILITY}

Supply chain visibility has different definitions in the literature [40]. Visibility in the supply chain refers to the ability of supply chain parties to access timely and accurate key information [7]. In the work of Simatupang and Sridharan [41], the authors defined supply chain visibility as: "The ability to trace the points of origin of materials used in a product". It is widely agreed that the limited visibility that companies have over their supply significantly contributes to lowering the performance of supply chains $[3,7,16]$. Since poor visibility on supply chains has negative effects such as long lead times [42], counterfeiting [16], stock-outs situations [43], bullwhipeffect[44] and high inventory cost. Although several efforts have been made in the area to improve the poor visibility issue[5], still poor visibility is a major challenge and has negative effects on supply chain networks performance $[8,30$, 45]. Table 1 summarizes some of the existing models that have been proposed for the poor visibility issue.

Reviewing the existing models that have been proposed to increase the supply chain visibility in Table 1 shows that recent models tend to utilize new emerging technologies such as FRID tags in [49], Cloud computing in [6], and Blockchain technology for the poor visibility issue supply chain in $[14,16$, 30, 45-48]. However, these models suffer from technological limitations in the technology adoption. For example, some of the presented solutions built their models on public Blockchain such as [14, 48]. In this case, the limitations of public Blockchain apply to these models such as: technical difficulties faced by miners (the network participants in Blockchain public networks) and the rapidly growth of blocks size.

TABLE I. SuPPly Chain VisibiLITy EXISTING Models

\begin{tabular}{|l|l|l|}
\hline Author & Year & \multicolumn{1}{c|}{ Solution } \\
\hline$[6]$ & 2018 & Cloud-based information sharing \\
\hline$[30]$ & 2018 & Hybrid private/public ledger based on Blockchain \\
\hline$[3]$ & 2018 & Supplier Development \\
\hline$[46]$ & 2018 & A decentralized information sharing approach \\
\hline$[47]$ & 2018 & HACCP, Blockchain and IoT traceability system \\
\hline$[45]$ & 2018 & Blockchain-based food traceability system \\
\hline$[16]$ & 2017 & Distributed data-driven application \\
\hline$[14]$ & 2017 & Online shipment tracking framework \\
\hline$[48]$ & 2017 & Information sharing Blockchain scheme \\
\hline$[49]$ & 2016 & Automatic identification (Auto-ID) technologies \\
\hline$[40]$ & 2016 & Suppliers selection \\
\hline
\end{tabular}


Although number of studies addressed the importance of supply chain visibility, there is a lack of common effective evaluation metrics to measure supply chain visibility [7, 43]. Visibility measurement is the degree of information sharing within the supply chain [10]. Some scholars in the relevant literature such as [50] and [51] emphasized on the traceability to measure the supply chain visibility. While another stream of visibility measurement work such as [52] pointed to the inventory visibility as a critical measure for supply chain visibility. Additionally, many studies have been proposed to improve the performance of the supply chain. However, the performance still needs to improve. Due to the increasing complexity of supply chains over the past years, many factors significantly contributes to lowering the performance of supply chains $[8,53]$ such factors as:

- Lack of information sharing [6].

- Poor demand and inventory visibility [8].

- Inventory information inaccuracy [54].

- Information leakage [55].

- Out-of-stock situation [54].

- Poor visibility [3], among others.

Likewise, several models have been developed to improve the visibility of supply chains. However, poor visibility is still a major challenge in complex supply chain network which lowers the supply chains performance $[3,8,30]$. Additionally, poor visibility has negative effects such as counterfeiting [16], stock-outs [43], and bullwhip-effect [7, 44]. Furthermore, this issue is considered as an obstacle to efficient SCPM [8]. Therefore, increasing supply chain visibility helps in improving the business performance since the visibility feature provides an access to accurate information that is necessary for decision-making [14]. As a consequence, a higher level of production, facilitate order traceability, and inventory information visibility will be obtained $[6,10]$.

Failing short to consider the poor visibility issue leads to inefficient business processes due to lack of information sharing, un seen supply and demand forecast, inventory inaccuracy. As a consequent, leads to low supply chain performance. Therefore, this study is an attempt to fill this gap by proposing supply chain network model based on Blockchain technology smart contract to increase the visibility measurement properties (information sharing, traceability, and inventory visibility).

\section{RESEARCH AGENDA}

The author extensively reviews the potential of the appropriate technology for the poor visibility issue in supply chain. Arguably, it is largely agreed between academia and practitioners that the adoption of Blockchain technology is promising for information sharing, traceability and inventory visibility in supply chain $[14,16,17]$. Therefore, based on the reviewed literature recommendations, in this research a supply chain network model is proposed based on Blockchain technology smart contract to improve the visibility in supply chain networks. The proposed model uses the Blockchain smart contract to increase the information sharing; orders traceability; and inventory visibility within a supply chains network.

As the proposed model focuses on improving the visibility in term of improvement in information sharing, traceability and inventory visibility, this research work was designed to include three main phases in order to achieve this aim as depicted in Figure 2.

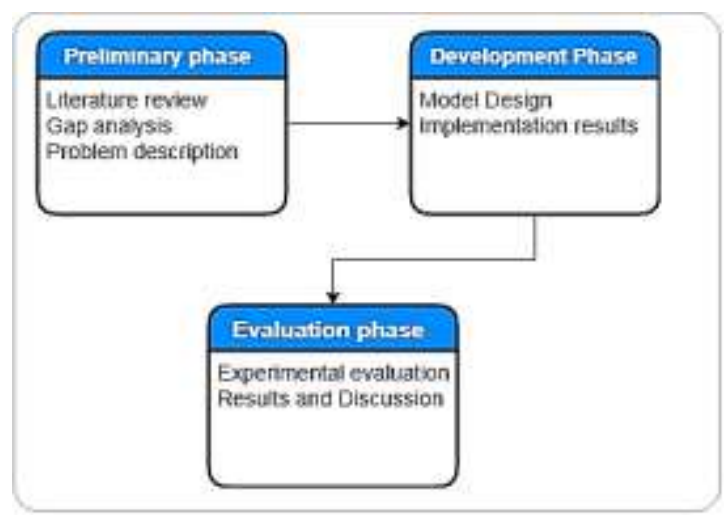

Fig. 2. Research Design.

The first phase: the preliminary phase which illustrates how the researcher identified the existing challenges in supply chain domain, influencing factors, knowledge gap, research problem, and the most appropriate technology to solve the specified research problem. In this phase, the supply chain management literature has been studied to explore these issues. This extensive literature review highlights the current existing challenges in the area and the existing knowledge gap. The second phase: the design and implementation phase where the development steps of the research model are proposed. The third phase: the evaluation phase which finalizes the research design. This phase aims to evaluate the proposed model in terms of improving the supply chain visibility. It represents the experimental evaluation results and the comparison results between the proposed (HCSC-POS) model, Cloud-based model and the traditional supply chain model.

\section{THE PROPOSED MODEL}

The proposed model is a Blockchain network model (HCSC-POS) improving the visibility in term of improvement in information sharing, traceability and inventory visibility. The proposed model is an extension and built based on Hyperledger Composer Supply Chain Network (HCSC) model which is an implementation of Blockchain platform "Hyperledger Composer" for the supply chains.

The proposed network model consists of three main layers: the business layer, Blockchain layer, and data layer. The first layer is the supply chain business layer which contains fourechelon supply chain trading participants:(Supplier, Manufacturer, Distributor, Retailer) and Customer. Herein, the supply chain participants are connected in a permissioned distributed immutable ledger built on top of HCSC network. Communication between network participants are in collaborative manner between upstream and downstream participants as shown in Figure 3. The second layer is the 
Blockchain layer. Wherein, this layer contains the necessary components and features to connect to the Blockchain such as the smart contract and the access control rules. This is the middle layer that connect the business layer with the data layer. The third layer is the data layer. This layer contains the data structure used in the proposed (HCSC-POS) network model. It contains the supply chain participants data, assets data, transactions and events data.

The supply chain network participants trading on a single asset, a product or "Commodity" and six transactions (InitiatePO ViewCommodity Order_Tracking TransferCommodity SetupDemo viewStock). In addition to three main events: StockLevels ProductDetails TrackOrders. The data structure of the proposed HCSC-POS network model consists of three main executable transaction processor functions that represent the smart contract. The subsequent sections will describe the structure and design of the transaction processor functions in details.

\section{A. ViewCommodity Transaction}

To achieve product visibility into the proposed (HCSCPOS) network model, the ViewCommodity transaction aims to share product information between HCSC-POS network participants. Throughout the following steps, network participants are able to view products, products origin, and the environmental profile of a product:

- Step1: the product owner posts to the Blockchain the following:

- Product details (tradingSymbol, name, description, origin, quantity, unitPrice, totalPrice, Environmental_Profile, SaftyStock StockLevelNotification, purchaseOrder, owner, issuer of purschase order), Product origin, Environmental profile.

- Step 2: the issuer/buyer submits ViewCommodity transaction uses the tradingSymbol of the desired product.

- Step 3: If the transaction is successful, the issuer/buyer is provided with full visibility of product details, origin and the environmental profile as Figure 4 shows the flow of information between network participants when submitting ViewCommodity transaction.

Herein, product information is accessible to relevant parties in HCSC-POS network. Customers are now aware of the product origin, and the environmental profile of the product before they buy or place a purchase order of this product.

\section{B. Order_Tracking Transaction}

Tracking orders transaction gives the ability to track an order location and status by the order issuer. When a supplier purchases a product, the order status changes to "initiated" purchase order. After confirming the purchase order, the order status changes to "Confirmed" and the order will be added to the ledger. Then, the vendor performs a TransferCommodity transaction to transfer the ordered product ownership from product owner to the new product owner. The steps followed in order tracking are as follows and shown in Figure 5:
- Step1: The vendor posts to the ledger the current status of the order, "Trace" values and order information.

- Step2: The unique orderId property is submitted to Order_Tracking transaction by the orderer.

- Step3: The associated event 'TrackOrders' pushes Trace values, order information and the current order status which promotes orders traceability.

The execution of Order_Tracking transaction pushes the trace information (timestamp, shipperLocation, and Trader company) to the assets registry. The transaction emits event 'TrackOrders' which locates the order tracking information as depicted in Figure 5.

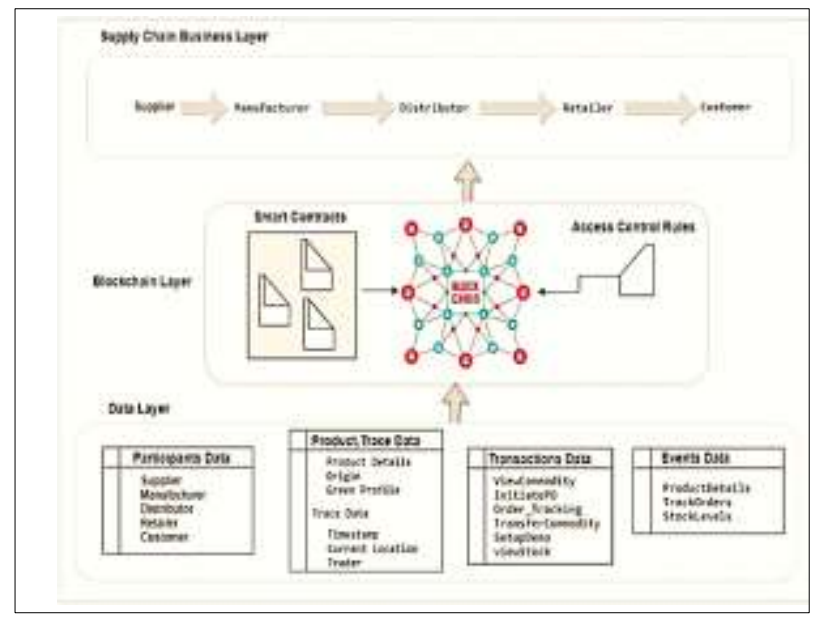

Fig. 3. The Proposed (HCSC-POS) Network Model.

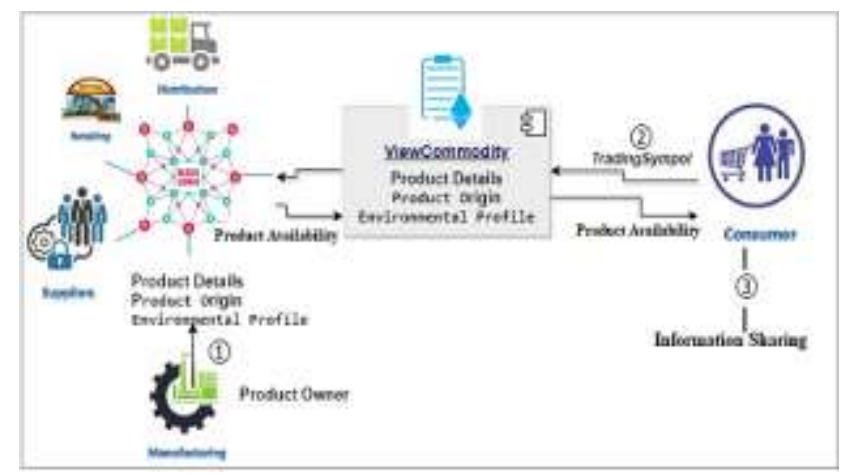

Fig. 4. Transaction View Commodity Flow.

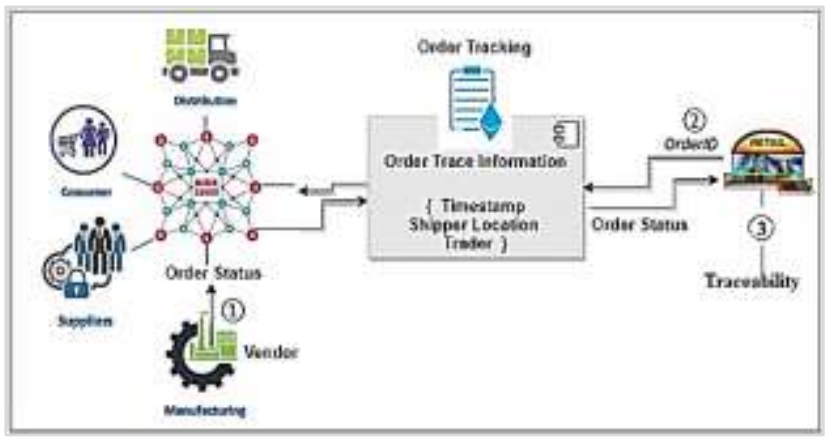

Fig. 5. Transaction Order Tracking Flow. 


\section{ViewStock Transaction}

In order to manage the inventory of the supply chain participants in HCSC-POS network model, the viewStock transaction queries the available quantity in stock for certain products using the tradingSymbol identifier. Then calculates the safety stock based on DailySalesUnits, and leadtime values. The safety stock in HCSC-POS network model is calculated based on the standard safety stocks equation in supply chain inventory theory is adopted from [56]:

SafetyStock $=($ MaximumDailyUsage $\times$ MaximumLeadTime $)-$

(AverageDailyUsage $\times$ AverageLeadTime )

The transaction compares the stock quantity with the safety stock value. If the safety stock level is reached, automatically notify the participant to place a purchase order for that product. Afterward, the product ownership will be changed to the new owner of the product accordingly.

- Step1: Calculates the safety stock.

- Step2: Check product quantity in stock using product tradingSymbol.

Step3: If product quantity reached the safety stock level, emit event StockLevels and notify product owner by notification message: "Product quantity is less than the safety stock levels "to place purchase order for this product and change the product ownership to the new owner of the product accordingly. Otherwise, if product quantity not reached the safety stock level yet, notification message: "Product quantity within safety stock levels "Figure 6 shows the flow of viewStock transaction.

Product quantity always updated with the most recent, current and available quantity in the stock. This is especially true after performing a purchase order and deduce the ordered quantity from the available quantity in the stock.

Comparison between the updated quantity in stock and the SaftyStock value, if the available quantity is less than or equals to the safety stock, a stock level notification message will be pushed to notify the vendor with "This product has reached the safety stock levels" notification message. Event 'StockLevels' emits and posts Product, oldQty, newQty, SaftyStock to the ledger. This event allows full visibility on information related to inventory levels.

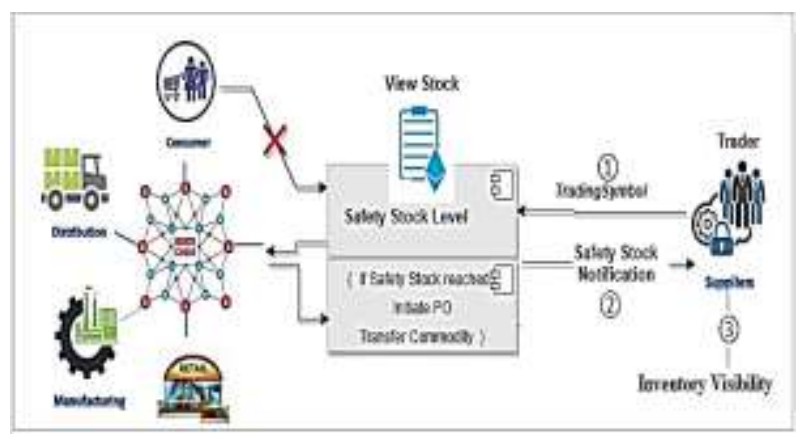

Fig. 6. Transaction viewStock Flow.

\section{Performance Evaluation}

In the evaluation phase, performance evaluation will be conducted. The goal is to evaluate the visibility improvement in the proposed HCSC-POS model in terms of information sharing, traceability and inventory visibility.

Therefore, the proposed model will be compared to Cloudbased information system in Gonul Kochan, Nowicki [6], and the traditional supply chain in term of reduction in lead times and inventory levels. Lead time and average inventory levels are common performance metrics that are reduced when visibility is improved in the supply chain [43]. These performance metrics were used in [6] to evaluate the performance of the proposed model. This process is shown at Figure 7.

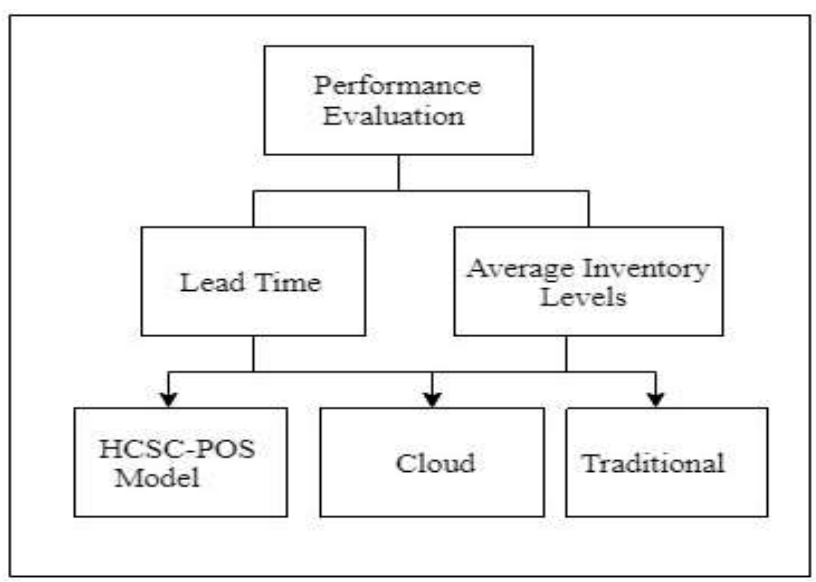

Fig. 7. Performance Evaluation.

In the first comparison, HCSC-POS network model will be compared against the cloud-based information system and the traditional supply chain model, whereby the lead time in HCSC-POS model is compared to the lead time in each model. The second part of the comparison will compare the average inventory levels in HCSC-POS, cloud-based, and the traditional supply chain model as shown in Figure 7. The goal is to evaluate the proposed HCSC-POS model in terms of improving the supply chain visibility bases on the specified common performance metrics.

\section{CONCLUSION}

Poor visibility is a major challenge in supply chain networks as it is considered as an obstacle to efficient supply chain performance. Although prior studies have dealt with the issue, however, still poor visibility under an increasingly complex supply chain networks environment a major challenge for supply chain executives. Additionally, although new emerging technologies significantly contribute to overcome the poor visibility issue. Still, some limitations have been reported in the adoption of these technologies.

This paper proposes supply chain network model to improve the supply chain visibility using Blockchain technology smart contract. The proposed model focuses on improving the visibility in term of improvement in the visibility measurement properties: information sharing, traceability and inventory visibility. The information sharing 
and traceability platform offer an end-to-end visibility of products provenance, orders statuses, raw material origins, and identifying green products which facilitates the access to key information in the supply chain network that is necessary for decision making. The ability to track orders provides accessibility to trace information. While inventory visibility enables supply chain participant to better control over their inventory by providing accurate inventory updates. As a result, preventing stock repetition, preventing out of stock situation, and bullwhip-effect and reducing the lead times and safety stock level which is accordingly reduces the inventory cost. The benefits that offered by the proposed model are essential criteria for competitiveness, necessary to support critical business decisions, and overall leads to supply chain performance improvements. Future researches could extend HCSC-POS model to include assessment of more performance metric measurements. Additionally, quantifying the visibility and including the sustainability dimensions (environmental, social, and financial) in the HCSC-POS model to solve environmental challenges could be fruitful research areas.

\section{ACKNOWLEDGMENT}

The research reported in this study is conducted by the researchers at Universiti Malaysia Pahang (UMP), it is funded by RDU1903111 and PGRS1903188 grants. The researchers would like to thank UMP for supporting this research.

\section{REFERENCES}

[1] Hofmann, H., et al., Sustainability-related supply chain risks: conceptualization and management. Business Strategy and the Environment, 2014. 23(3): p. 160-172.

[2] Giannakis, M. and T. Papadopoulos, Supply chain sustainability: A risk management approach. International Journal of Production Economics, 2016. 171: p. 455-470.

[3] Pradhan, S.K. and S. Routroy, Improving supply chain performance by Supplier Development program through enhanced visibility. Materials Today: Proceedings, 2018. 5(2): p. 3629-3638.

[4] Rostamzadeh, R., et al., Evaluation of sustainable supply chain risk management using an integrated fuzzy TOPSIS- CRITIC approach. Journal of Cleaner Production, 2018. 175: p. 651-669.

[5] Swift, C., V.D.R. Guide Jr, and S. Muthulingam, Does supply chain visibility affect operating performance? Evidence from conflict minerals disclosures. Journal of Operations Management, 2019.

[6] Gonul Kochan, C., et al., Impact of cloud-based information sharing on hospital supply chain performance: A system dynamics framework. International Journal of Production Economics, 2018. 195: p. 168-185.

[7] Somapa, S., M. Cools, and W. Dullaert, Characterizing supply chain visibility-a literature review. The International Journal of Logistics Management, 2018. 29(1): p. 308-339.

[8] Dweekat, A.J., G. Hwang, and J. Park, A supply chain performance measurement approach using the internet of things: Toward more practical SCPMS. Industrial Management \& Data Systems, 2017. 117(2): p. 267-286.

[9] Messina, D., A.C. Barros, and A. Lucas, How much visibility has a company over its supply chain? A diagnostic metric to assess supply chain visibility. 2018.

[10] Francisco, K. and D. Swanson, The Supply Chain Has No Clothes: Technology Adoption of Blockchain for Supply Chain Transparency. Logistics, 2018. 2(1): p. 2.

[11] Lehmacher, W., et al. Impact of the Fourth Industrial Revolution on Supply Chains. in World Economic Forum, Geneva, Switzerland, REF. 2017.

[12] Morris, V., et al., Developing a Blockchain Business Network with Hyperledger Composer using the IBM Blockchain Platform Starter Plan. International Technical Support Organization, 2018.
[13] Korpela, K., J. Hallikas, and T. Dahlberg. Digital supply chain transformation toward blockchain integration. in proceedings of the 50th Hawaii international conference on system sciences. 2017.

[14] Wu, H., et al., A Distributed Ledger for Supply Chain Physical Distribution Visibility. Information, 2017. 8(4): p. 137.

[15] Kshetri, N., 1 Blockchain's roles in meeting key supply chain management objectives. International Journal of Information Management, 2018. 39: p. 80-89.

[16] Madhwal, Y. and P.B. Panfilov, Blockchain and Supply Chain Management: Aircrafts'parts'business Case. Annals of Daaam and Proceedings, 2017. 28.

[17] Min, H., Blockchain technology for enhancing supply chain resilience. Business Horizons, 2019. 62(1): p. 35-45.

[18] Pournader, M., et al., Blockchain applications in supply chains, transport and logistics: a systematic review of the literature. International Journal of Production Research, 2019: p. 1-19.

[19] Saberi, S., et al., Blockchain technology and its relationships to sustainable supply chain management. International Journal of Production Research, 2019. 57(7): p. 2117-2135.

[20] Nakamoto, S., Bitcoin: A peer-to-peer electronic cash system. 2008.

[21] Risius, M. and K. Spohrer, A Blockchain Research Framework What We (don't) Know, Where We Go from Here, and How We Will Get There. Business \& Information Systems Engineering, 2017. 59(6): p. 385-409.

[22] Kamble, S., A. Gunasekaran, and H. Arha, Understanding the Blockchain technology adoption in supply chains-Indian context. International Journal of Production Research, 2019. 57(7): p. 2009-2033.

[23] Christidis, K. and M. Devetsikiotis, Blockchains and smart contracts for the internet of things. Ieee Access, 2016. 4: p. 2292-2303.

[24] Bünger, M. Blockchain for industrial enterprises: Hype, reality, obstacles and outlook. 2017 30-05-2018]; Available from: https://internetofthingsagenda.techtarget.com/blog/IoTAgenda/Blockchain-for-industrial-enterprises-Hype-reality-obstaclesand-outlook.

[25] Imbault, F., et al., The green blockchain Managing decentralized energy production and consumption. 2017 1st Ieee International Conference on Environment and Electrical Engineering and 2017 17th Ieee Industrial and Commercial Power Systems Europe (Eeeic / I\&Cps Europe), 2017.

[26] Dabbs, A. What blockchain can do for the environment. 2017 20-052018]; Available from: https://www.greenbiz.com/users/alistair-dabbs.

[27] Li, Z., et al. A Hybrid Blockchain Ledger for Supply Chain Visibility. in 2018 17th International Symposium on Parallel and Distributed Computing (ISPDC). 2018. IEEE.

[28] Kouhizadeh, M. and J. Sarkis, Blockchain practices, potentials, and perspectives in greening supply chains. Sustainability, 2018. 10(10): p. 3652

[29] Plant, L., Implications of open source blockchain for increasing efficiency and transparency of the digital content supply chain in the australian telecommunications and media industry. Australian Journal of Telecommunications and the Digital Economy, 2017. 5(3): p. 15.

[30] Li, Z., et al., A Hybrid Blockchain Ledger for Supply Chain Visibility. 2018: p. 118-125.

[31] Casino, F., T.K. Dasaklis, and C. Patsakis, A systematic literature review of blockchain-based applications: Current status, classification and open issues. Telematics and Informatics, 2019. 36: p. 55-81.

[32] Zyskind, G., O. Nathan, and A. Pentland, Decentralizing Privacy: Using Blockchain to Protect Personal Data. 2015 Ieee Security and Privacy Workshops (Spw), 2015: p. 180-184.

[33] Kosba, A., et al. Hawk: The blockchain model of cryptography and privacy-preserving smart contracts. in Security and Privacy (SP), 2016 IEEE Symposium on. 2016. IEEE.

[34] Tian, F. An agri-food supply chain traceability system for China based on RFID \& blockchain technology. in Service Systems and Service Management (ICSSSM), 2016 13th International Conference on. 2016. IEEE.

[35] Kshetri, N., Can blockchain strengthen the internet of things? IT professional, 2017. 19(4): p. 68-72. 
[36] Omran, Y., et al., Blockchain-driven supply chain finance: Towards a conceptual framework from a buyer perspective. 2017.

[37] Galvez, J.F., J. Mejuto, and J. Simal-Gandara, Future challenges on the use of blockchain for food traceability analysis. TrAC Trends in Analytical Chemistry, 2018. 107: p. 222-232.

[38] Dolgui, A., et al., Blockchain-oriented dynamic modelling of smart contract design and execution in the supply chain. International Journal of Production Research, 2019: p. 1-16.

[39] Queiroz, M.M., R. Telles, and S.H. Bonilla, Blockchain and supply chain management integration: A systematic review of the literature. Supply Chain Management: An International Journal, 2019.

[40] Yousefi, S., H. Mahmoudzadeh, and M. Jahangoshai Rezaee, Using supply chain visibility and cost for supplier selection: a mathematical model. International Journal of Management Science and Engineering Management, 2017. 12(3): p. 196-205.

[41] Simatupang, T.M. and R. Sridharan. A characterization of information sharing in supply chains. in ORSNZ Conference University of Canterbury, New Zealand. 2001.

[42] Apiyo, R. and D. Kiarie, Role of ICT tools in supply chain performance International Journal of Supply Chain Management, 2018. 3(1): p. 17-26.

[43] Caridi, M., et al., Measuring visibility to improve supply chain performance: a quantitative approach. Benchmarking: An International Journal, 2010. 17(4): p. 593-615.

[44] Barratt, M. and R. Barratt, Exploring internal and external supply chain linkages: Evidence from the field. Journal of Operations Management, 2011. 29(5): p. 514-528.

[45] Mohan, T., Improve Food Supply Chain Traceability using Blockchain. 2018, The Pennsylvania State University.

[46] Imeri, A. and D. Khadraoui. The security and traceability of shared information in the process of transportation of dangerous goods. in New Technologies, Mobility and Security (NTMS), 2018 9th IFIP International Conference on. 2018. IEEE.
[47] Tian, F., An information System for Food Safety Monitoring in Supply Chains based on HACCP, Blockchain and Internet of Things. 2018, WU Vienna University of Economics and Business.

[48] Nakasumi, M. Information sharing for supply chain management based on block chain technology. in 2017 IEEE 19th Conference on Business Informatics (CBI). 2017. IEEE.

[49] Papert, M., P. Rimpler, and A. Pflaum, Enhancing supply chain visibility in a pharmaceutical supply chain: Solutions based on automatic identification technology. International Journal of Physical Distribution \& Logistics Management, 2016. 46(9): p. 859-884.

[50] Mattoli, V., et al., Flexible tag datalogger for food logistics. Sensors and Actuators A: Physical, 2010. 162(2): p. 316-323.

[51] Amr, M., et al. Merging supply chain and Blockchain technologies. in The International Maritime Transport and Logistics Conference, Marlog. 2019.

[52] Zhang, A.N., M. Goh, and F. Meng, Conceptual modelling for supply chain inventory visibility. International Journal of Production Economics, 2011. 133(2): p. 578-585.

[53] Lima-Junior, F.R. and L.C.R. Carpinetti, Quantitative models for supply chain performance evaluation: A literature review. Computers \& Industrial Engineering, 2017. 113: p. 333-346.

[54] Cui, L., et al., Investigation of RFID investment in a single retailer twosupplier supply chain with random demand to decrease inventory inaccuracy. Journal of cleaner production, 2017. 142: p. 2028-2044.

[55] Hassan, A.Y. and H.H. Nasereddin, Information Sharing Characteristics In Supply Chain Management. EPH-International Journal of Business \& Management Science (ISSN: 2208-2190), 2018. 4(1): p. 01-09.

[56] Chan, F., S. Routroy, and R. Kodali, Differential evolution algorithm for supply chain inventory planning. Journal of Manufacturing Technology Management, 2005. 